\title{
Duracon Total Knee Replacement in Kuwait: A Clinical and Radiographic Analysis of 52 Knees Followed for 2-5 Years
}

\author{
Ahmad Al-Rowaih ${ }^{a}$ Wieslaw Pospula ${ }^{a}$ Osama Abdul-Moez ${ }^{\mathrm{a}}$ \\ Anders Lindstrand ${ }^{\mathrm{b}}$ \\ aA Razi Orthopedic Hospital, Kuwait; bDepartment of Orthopedics, University Hospital, \\ Lund, Sweden
}

\section{Key Words}

Total knee arthroplasty · Osteoarthrosis • Rheumatoid arthritis · Hospital for Special Surgery score $\cdot$ Mechanical axis · Outcome

\begin{abstract}
Objective: In this prospective clinical and radiological study results of 52 consecutive Duracon bicompartmental total knee arthroplasties in 37 patients with femorotibial arthrosis and rheumatoid arthritis with the average observation time of 3 (2-5) years were analyzed. Methods: The study includes 32 women (44 knees) and 5 men (8 knees) with a mean age of 60 years. Details of implant design and surgical technique were given. Two surgeons performed all the operations. The patients were evaluated by an unbiased observer. The Hospital for Special Surgery (HSS) score for both pre- and postoperative assessment was used. Results: The average initial HSS score of 44 (20-65) improved to 85 (60-99) after operation. By subjective assess-
\end{abstract}

\begin{tabular}{ll}
\hline KARGER & $\begin{array}{l}\text { (1) 1999 S. Karger AG, Basel } \\
\text { 1011-7571/99/0082-0098\$17.50/0 }\end{array}$ \\
$\begin{array}{l}\text { Fax + 41 61 306 1234 } \\
\text { E-Mail karger@karger.ch } \\
\text { www.karger.com }\end{array}$ & $\begin{array}{l}\text { Accessible online at: } \\
\text { http://BioMedNet.com/karger }\end{array}$
\end{tabular}

ment, 41 knees were excellent, 10 good and 1 knee had a fair result. There were no revisions. The mean postoperative flexion was $101^{\circ}(80-120)$. The mean anteroposterior angle of the tibial component was $4^{\circ}$ of the varus and the mean lateral angle was $3^{\circ}$ of the posterior slope. The mean postoperative hip-knee-ankle deviation was $4^{\circ}$ of the varus. Conclusion: In Kuwait, the cemented Duracon total knee arthroplasty without patellar resurfacing gave satisfactory clinical results after an intermediate follow-up period of 3 years.

\section{Introduction}

The results of total knee arthroplasties have been increasingly successful during the last 25 years [1-4]. Outcome studies as well as national registers have shown a high patient satisfaction and a gradual lowering of the cumulative revision frequency [5-7]. The de- 
velopment of knee prosthesis design has a long history and is marked by the Massachusetts General Hospital design of femoral resurfacing, the McIntosh tibial plateau and Marmor Compartmental designs. There were also hinged prostheses of which the Swedish design of Dr. Walldius and a French group, Guepar, were the most remarkable developmental steps. These early prosthetic types were gradually replaced by more modern, less constrained devices and today's different types of total knee arthroplasty are of resurfacing bi- or tricompartmental type. Especially during the 1990s, satisfactory results have been reported also for younger [8-10] and heavier [11-13] patients.

\section{Patients and Methods}

Fifty-two consecutive Duracon bicompartmental total knee arthroplasties in 37 patients, performed during the period 1992 through 1995, were prospectively collected and followed. All operations were performed by 2 surgeons. There were 32 females ( 44 knees) and 5 males ( 8 knees). Thirty-five patients ( 48 knees) were Kuwaitis and the remaining 3 patients ( 4 knees) were of other Arab nationalities. The mean age at the time of surgery was $60(25-76)$ years. Thirty-three patients (46 knees) had femorotibial arthrosis (OA) stages II-V $[14]$ engaging the medial $(n=45)$ or lateral $(n=1)$ compartment, respectively. The remaining 4 patients $(6$ knees) had rheumatoid arthritis (RA, table 1). The stage of the OA disease was often advanced and 21 knees had Ahlbäck stage IV and 13 stage V. In all knees pre- and postoperative standing radiographs were performed as well as long standing hip-knee-ankle (HKA) radiographs. The preoperative standing long leg radiograph $(n=46)$ showed in mean a deviation of the mechanical axis (HKA) of $14^{\circ}$ varus (range $25^{\circ}$ varus to $5^{\circ}$ valgus). In the remaining 6 knees, there was a marked fixed flexion deformity, and therefore a standing long leg radiograph was not possible to properly obtain and measure. Preoperatively the patients had disabling pain on weight bearing and 1 in 2 had also pain at rest. The weight was in mean $80(65-100) \mathrm{kg}$ and the height $159(150-172) \mathrm{cm}$. The Hospital Special Surgery (HSS) score [15] evaluating pain, function, mobility, muscle strength, flexion deformity and insta-

Duracon Total Knee Replacement in Kuwait
Table 1. Age, sex, diagnosis, and follow-up

\begin{tabular}{llc}
\hline Age, years & Mean & 60 \\
& Range & $25-76$ \\
Sex & Male & 5 (8 knees $)$ \\
Diagnosis & Female & 32 (44 knees) \\
& OA & 33 (46 knees) \\
Follow-up, years & RA & 4 (6 knees $)$ \\
& Mean & 3 \\
& Range & $2-5$ \\
\hline
\end{tabular}

bility was used for the clinical pre- and postoperative comparison. The mean preoperative HSS score was 44 (20-62). Sixteen patients ( 25 knees) had other significant health problems like diabetes, hypertension or coronary heart disease. An unbiased observer, who was not performing the surgical procedure and who was not involved in the regular follow-up of the patients, performed the postoperative clinical assessment and evaluation of the radiographs.

\section{Implant}

The Duracon total knee arthroplasty was used as a cemented bicompartmental replacement, which means that distal femur and proximal tibia were replaced by an artificial implant. Although the design of the prosthesis includes a patellar button, the authors deliberately did not replace the patella as a rule in this series of patients. The femoral component was composed of a metal alloy consisting of chrome, cobalt and molybdenum while the tibial component has the same metal as a base while the bearing surface was a polymer consisting of ultra-high molecular weight polyethylene (fig. 1). Both the femoral and tibial component have six different options of sizes to match individual differences in dimensions. There were seven different variations in polyethylene thickness, from 7 to $21 \mathrm{~mm}$, in order to achieve optimal stability of the arthroplasty. Both the femoral and the tibial component were fixed with polymethylmethacrylate bone cement to anchor the implant to bone. Porous coated implants with cement were used in this study as it appeared difficult to achieve tight fit for uncemented use. For uniformity of the study this was continued till the end.

\section{Operative Technique}

The operation was performed under aseptic conditions and always with prophylactic antibiotics. A mid- 
Fig. 1. General view of prosthetic components.

Fig. 2. Intraoperative view of implanted prosthesis.
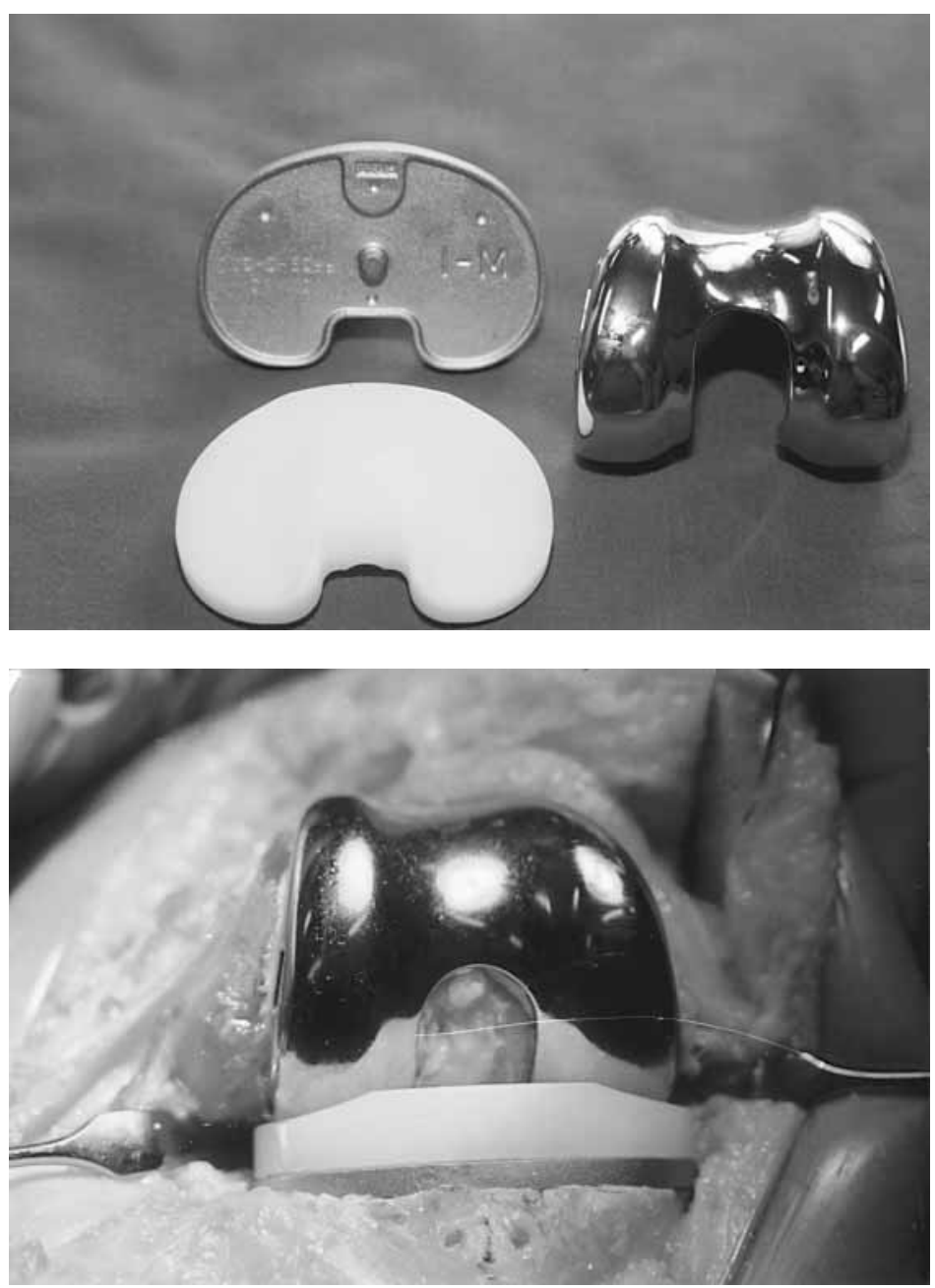

line skin incision was used followed by a parapatellar medial arthrotomy. First, any osteophytes were removed from the condyles and patella. Then the first generation of the universal guide instruments was used in order to control the resections and drillings. Classical alignment technique was used with the intramedullary femoral guiding system and extramedullary tibias guiding system. As a rule the posterior cruciate ligament was spared. In patients with marked fixed flexion deformity posterior release with partial or complete release of the posterior cruciate ligament was done. On the tibial side condylar insert was used. The different jigs were sequentially used and the cuts were checked for inclination and surface evenness. Bone cement with gentamycin (Palacos) was always used when in low viscosity state for improved penetration into the bone as well as the porous coating of the component (fig. 2-4). For better bone anchorage, multiple holes in different directions were drilled especially where the underlying bone was sclerotic. Cement-gun pressurization or pulsative lavage were not used. Eight extra small, 33 small, 7 medium, 4 medium-large femoral components were used. On the tibial side 18 small, 26 medium and 4 medium-large and 4 large components were used. The height of the tibial component was $9 \mathrm{~mm}$ in $18 \mathrm{knees}, 11 \mathrm{~mm}$ in 29 knees and $13 \mathrm{~mm}$ in 5 knees. 
3

Fig. 3. X-ray picture of implanted prosthesis, AP view.

Fig. 4. X-ray picture of implanted prosthesis, lateral view.

Table 2. Mean values of preoperative and postoperative clinical assessment

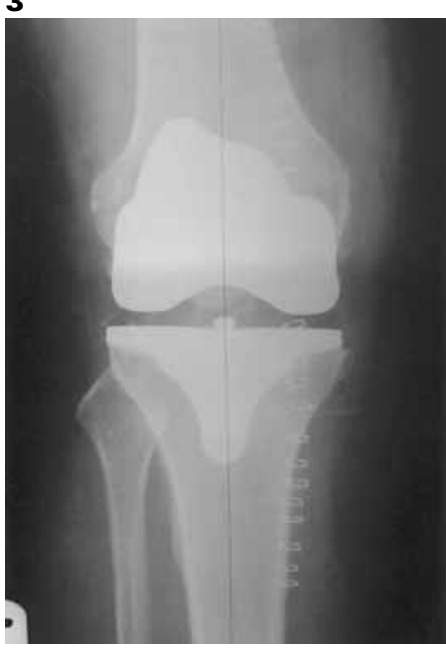

4

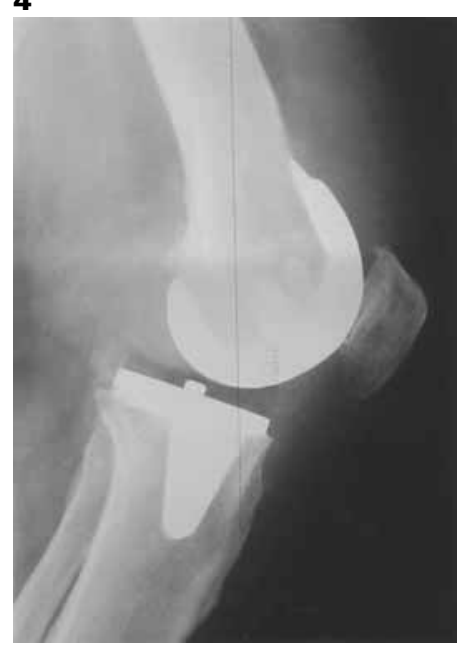

Fix. fl. def. $=$ Fixed flexion deformity

\section{Results}

The follow-up time was in mean 3 (2-5) years. By subjective assessment, 41 knees were excellent, 10 good while 1 knee was classified as fair. By objective assessment the mean postoperative HSS score was, at the last follow-up, 85 (60-99) with 37 excellent, 13 good and 2 fair. The results of certain different HSS parameters are shown in table 2. There were no revisions performed. One operation was performed in a patient with RA and myasthenia gravis, who developed a deep he- matogenous salmonella infection in the replaced knee joint 1.5 years after the index operation. An arthrotomy and a concomitant debridement were done and 1 year after this procedure the patient was free of symptoms and signs of infection. Blood tests and indium-labeled leukocyte scan were negative. Two other patients got superficial infections which subsided on antibiotics. One patient got a myocardial infarction during the hospital stay without any late consequences. The anteroposterior angle of the tibial component was in mean $4^{\circ}$ varus $\left(78^{\circ}-94^{\circ}\right)$ and the mean 
Table 3. Preoperative and postoperative radiological assessment

\begin{tabular}{|c|c|c|}
\hline $\begin{array}{l}\text { Radiological } \\
\text { parameters }\end{array}$ & Preoperative & Postoperative \\
\hline \multicolumn{3}{|c|}{ OA stage (Ahlbäck) } \\
\hline Stage 2 & 2 knees & \\
\hline Stage 3 & 16 knees & \\
\hline Stage 4 & 21 knees & \\
\hline Stage 5 & 13 knees & \\
\hline HKA angle & $\begin{array}{l}14^{\circ} \text { varus } \\
\left(25^{\circ} \text { varus to } 5^{\circ} \text { valgus }\right)\end{array}$ & $\begin{array}{l}4^{\circ} \\
\left(18^{\circ} \text { varus to } 6^{\circ} \text { valgus }\right)\end{array}$ \\
\hline \multicolumn{3}{|c|}{ Inclination of tibial component of the prosthesis } \\
\hline Anteropost & & $86(78-94)$ \\
\hline Lateral vie & & $86(80-92)$ \\
\hline
\end{tabular}

lateral angle was $4^{\circ}$ posterior slope (8092). There were no significant radiolucencies $(>1 \mathrm{~mm})$. Preoperative and postoperative radiological findings are summarized in table 3.

\section{Discussion}

The major evolution of arthroplasty of human knee joints has been continuing for more than 20 years [7]. The initial period had certain problems, which required frequent revision procedures of different types. Today, in the mature era of improved design principles, better material properties, more controlled operative technique as well as better knowledge about patient selection, the results are more predictable. In Kuwait, knee arthroplasty was introduced in 1984 and during the 1990s an increase of its use has taken place. Retrospectively, it was an advantage to wait until this technology was more controlled and the early problems were solved. The material described here pertains to the first, prospectively collected, consecutive series of total knee arthroplasties in the country. These very early results are satisfactory and equal to other series. Even if the follow-up time is short we believe it is justified to report this material now, as to the best of our knowledge, there are almost no similar reports with this study design from this part of the world. Also referring doctors in the region need information about this treatment program.

Usually the patients present themselves late for surgery. They try, for a long time, many nonoperative treatment programs first [16]. Therefore, it is not surprising that about half of the patients had the most advanced grade of the OA disease with a major wear of cartilage and bone, secondary subluxation of the joint leading to substantial limb deformity and subsequently a low preoperative function. The medial femorotibial OA was very common here, and it may have a relation to the frequently used Arabic sitting position. In this position, the medial femorotibial compartment is constantly under pressure, and possibly the wear will increase in this part of the joint by time. In this report there were 32 women and 5 men operated by total knee replacement. The female preponderance is even more clear than in previous reports [1-4] and may represent sex differences in the OA disease prevalence in this part of the world. The mean age of our patients is slightly lower

$\overline{102} \quad \overline{\text { Med Principles Pract 1999;8:98-104 }}$ 
compared to other series [3, 4] and the relation between height and weight indicates more overweight in our series [1,2]. However, there are reports that total knee arthroplasty is a reliable and durable procedure also in younger [8-10] and heavier [11-13] patients, especially if they have a sedentary life-style [9]. Lower functional demand is a major reason why we believe that the majority of our cemented total knee arthroplasties will last for a reasonably long time period. Patients with excessive overweight are, however, at a higher risk of failure due to loosening [2].

The HSS score was used [15]. The score gives a maximum of 30 points for no pain on walking or at rest, 22 points for a good function, 18 points for full range of motion, 10 points each for good muscle strength, no flexion deformity and no instability. Our patients had almost the same pre- and postoperative flexion. The postoperative flexion is equal to other published series [1, 2]. Our mean postoperative score of 85 can be explained by lower functional demands. Even if the patients are pain-free, our elderly patients usually walk only indoors and, if they are outdoors, they use transportation aid. Another circumstance affecting the HSS score is the general health of our patients where almost half of them had other significant diseases, which influenced their activity level. Our radiographic results show a small remaining varus position of the tibial component and for the arthroplasty. This is not uncommon in other series [17, 18]. Our patients had frequently the most advanced stages of the OA disease, which makes surgery more demanding. One other reason is that OA also affects lower limb torsion [19]. Yagi [19] showed that the normal $23.5^{\circ}$ of external tibial torsion was changed in OA stages IV and $\mathrm{V}$ to $7.5^{\circ}$ of external tibial torsion. Therefore, during surgery in certain knees, a compromise between the anteroposterior and the lateral alignment of the knee and the torsional alignment of the tibia has to be done. We also believe that better guide instruments as well as improved operative experience can improve the surgical accuracy [20]. The outliers regarding component positioning and limb alignment are, however, at an increased risk of developing radiolucencies and clinical loosening by time [1, 2].

We deliberately did not replace the patella in any patient [21]. Despite this, there was no patellar pain affecting the results. As kneeling during Arabic sitting and also during praying is common many times every day, failure is either due to loosening of the component or patellar fracture $[22,23]$. One proven infection occurred in a rheumatoid patient. This underlying disease has a higher risk of a postoperative infection than if the underlying disease is OA [7]. We are fully aware of the fact that the prosthesis is still at risk for a revision but presently the patient is doing well and there are no radiographic or other findings indicating a failure. 


\section{References}

1 Malkani AL, Rand JA, Bryan RS, Wallrichs SL: Total knee arthroplasty with the kinematic condylar prosthesis. J Bone Joint Surg 1995;77A: 423-431.

2 Ranawat CS, Flynn WF Jr, Saddler S, Hansraj KK, Maynard MJ: Longterm results of total condylar knee arthroplasty: A 15-year survivorship study. Clin Orthop 1993;286:94102.

3 Rand JA, Ilstrup DM: Survivorship analysis of total knee arthroplasty: Cumulative rates of survival of 9200 total knee arthroplasties. J Bone Joint Surg 1991;73A:397-409.

4 Scuderi GR, Insall JN, Windsor RE, Moran MC: Survivorship of cemented knee replacements. J Bone Joint Surg 1989;71B:798-803.

5 Knutson K, Lindstrand A, Lidgren L: Survival of knee arthroplasties: A nation-wide multicenter investigation of 8000 cases. J Bone Joint Surg Br 1986;68:795-803.

6 Callahan CM, Drake BG, Heck DA, Dittus RS, et al: Patient outcomes following tricompartmental total knee replacement: A meta-analysis. JAMA 1994;271:1349-1357.

7 Knutson K, Lewold S, Robertsson O, Lidgren L: The Swedish knee arthroplasty register: A nation-wide study of 30,003 knees 1976-1992. Acta Orthop Scand 1994;65:375386.
8 Ranawat CS, Padgett DE, Ohashi Y: Total knee arthroplasty for patients younger than 55 years. Clin Orthop 1989;248:27-33.

9 Stern SH, Bowen MK, Insall JN, Scuderi GR: Cemented total knee arthroplasty for gonarthrosis in patients 55 years old or younger. Clin Orthop 1990;260:124-129.

10 Diduch DR, Insall JN, Scott WN, Scuderi GR, Font-Rodriguez D: Total knee replacement in young, active patients: Long-term follow-up and functional outcome. $\mathbf{J}$ Bone Joint Surg 1997;79A:575-582.

11 Smith BE, Askew MJ, Gradisar IA Jr, Gradisar JS, Lew MM: The effect of patient weight on the functional outcome of total knee arthroplasty. Clin Orthop 1992;276:237-244.

12 Stern SH, Insall JN: Total knee arthroplasty in obese patients. J Bone Joint Surg 1990;72A:1400-1404.

13 Jiganti JJ, Goldstein WM, Williams CS: A comparison of the perioperative morbidity in total joint arthroplasty in the obese and nonobese patient. Clin Orthop 1993;289:175179.

14 Ahlbäck S: Osteoarthrosis of the knee: A radiographic investigation. Acta Radiol Diagn (Stockh) 1968 (suppl 277):7-72
15 Ranawat CS, Insall J, Shine J: Duocondylar knee arthroplasty: Hospital for special surgery design. Clin Orthop 1976;120:76-82.

16 Brown CR: Medical treatment of arthritis; in Callaghan JJ, et al (eds): Hip and Knee Reconstruction. American Academy of Orthopaedic Surgeons, 1995.

17 Ryd L: Micromotion of Knee Arthroplasty; thesis Lund, 1986.

18 Toksvig-Larsen S: On Bone Cutting; thesis Lund, 1992.

19 Yagi T: Tibial torsion in patients with medial-type osteoarthrotic knees. Clin Orthop 1994;302:5256.

20 Lewold S, Knutson K, Lidgren L: Reduced failure rate in knee prosthetic surgery with improved implantation technique. Clin Orthop 1993;287:94-97.

21 Levitsky KA, Harris WJ, McManus J, Scott RD: Total knee arthroplasty without patellar resurfacing: Clinical outcomes and long-term followup evaluation. Clin Orthop 1993; 286:116-121.

22 Dennis DA: Patellofemoral complications in total knee arthroplasty: A literature review. Am J Knee Surg 1992;5:156-166.

23 Kirk P, Rorabeck CH, Bourne RB, Burkart B, Nott L: Management of recurrent dislocation of the patella following total knee arthroplasty. J Arthroplasty 1992;7:229-233. 